\title{
Full scale simulation of a field-emitter arrays based electron source for free-electron lasers
}

\author{
M. Dehler ${ }^{\mathrm{a})}$ \\ Paul Scherrer Institut, CH-5232 Villigen PSI, Switzerland \\ A. Candel \\ ETH Zuerich, CH-8093 Zürich, Switzerland \\ E. Gjonaj \\ TU Darmstadt, D-64289 Darmstadt, Germany
}

(Received 9 November 2005; accepted 6 February 2006; published 27 March 2006)

\begin{abstract}
We describe the computer modeling of relativistic electron guns $(0.5 \mathrm{MeV})$ using pulsed field emitter arrays. The special challenge lies in the fact, that current distributions vary at a submicron scale, whereas structural dimensions are in the millimeter range. The general approach uses two steps. The first one is the computation of individual field-emitter tips including gate and focusing layers. Real world influences as, e.g., the effect of adsorbates on the emitted currents are taken into account by parameterizing the phase space of the tips. Together with a stochastic distribution of emitter properties, this leads to an equivalent current distribution on the cathode itself, which is used in the second step for the calculation of the electron dynamics in the gun itself. We present results for a source using a field-emitter array of 17700 tips. For the current field-emitter geometry, we see a large high base line effect resulting from nonlinear focusing forces inside the emitter itself. Of special interest are the effects of spatial correlations in the stochastic distribution on the emittance, showing pronounced performance degradations in the case of large correlation lengths. (C) 2006 American Vacuum Society. [DOI: 10.1116/1.2181988]
\end{abstract}

\section{INTRODUCTION}

The performance of well-designed electron linear accelerators is critically dependent on the situation inside the very first few millimeters of the accelerator, the electron gun. The electrons are still at nonrelativistic velocities and thus, a major part of beam quality deterioration is taking part in this location. A thorough understanding of the interactions there is required for the development of high performance designs.

PSI started a research project (Low Emittance Gun project or LEG) on a new generation of free-electron lasers with a special emphasis on high brilliant electron sources using field-emitter arrays (FEA). Apart from simulation described, it includes development work for pulsed high-voltage dc guns, production of field-emitter arrays as well as conceptual design studies on a free-electron laser.

A free-electron laser, as shown in Fig. 1 consists basically of a linear accelerator providing a high-intensity electron beam into an undulator section. The undulator, a magnet structure with an alternating transverse field, causes the beam to oscillate at the same time radiating synchrotron radiation. With the synchrotron radiation strong enough to act back on the beam causing a modulating of the charge density, a self amplifying effect, self-amplification by spontaneous emission (SASE), is obtained.

Critical in that respect is an intense and well-ordered beam, so that the particles follow synchronous trajectories within the undulator, avoiding phase cancellations of the radiated electromagnetic waves. As a consequence, we are

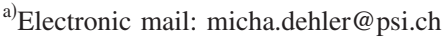

aiming for a very parallel beam with extremely small transverse momentum spread out of a field-emitter array, which gets frozen by rapid acceleration.

Here, field emitters may have generic advantages given their small emission surface and subsequent high current density. If these are pulsed via a gate nanosecond pulse the tips do not get into thermal equilibrium, so that they can be overloaded allowing even higher limits in the current density. This effect has already been demonstrated for pulses of $100 \mathrm{~ns}$ in Ref. 1. Protective techniques as using a resistive layer at the base of the tips should not be needed (and would not be welcome due to their negative influence on the bandwidth). The size of gate and other layers have to be restricted to the area of the emitter itself to minimize the gate to emitter capacitance. The generated beamlets are highly divergent, so they need to be individually focused using an additional focusing layer on top of the gate to combine them into a highly brilliant macroscopic beam with a correspondingly low momentum spread (Fig. 2). Assuming a total current of $5 \AA$ out of a FEA of $500 \mu \mathrm{m}$ diameter, the resulting beam is heavily space charge dominated, so that the beam parameters need to be frozen by a rapid acceleration to relativistic velocities. This is realized by putting the cathode into a pulsed diode configuration creating an accelerating field of $500 \mathrm{MV} / \mathrm{m}$.

\section{SIMULATION APPROACH}

Computing the fully detailed structure in one step is not feasible and not necessary. On the one hand, one would have to resolve simultaneously the nanometer size details of the 


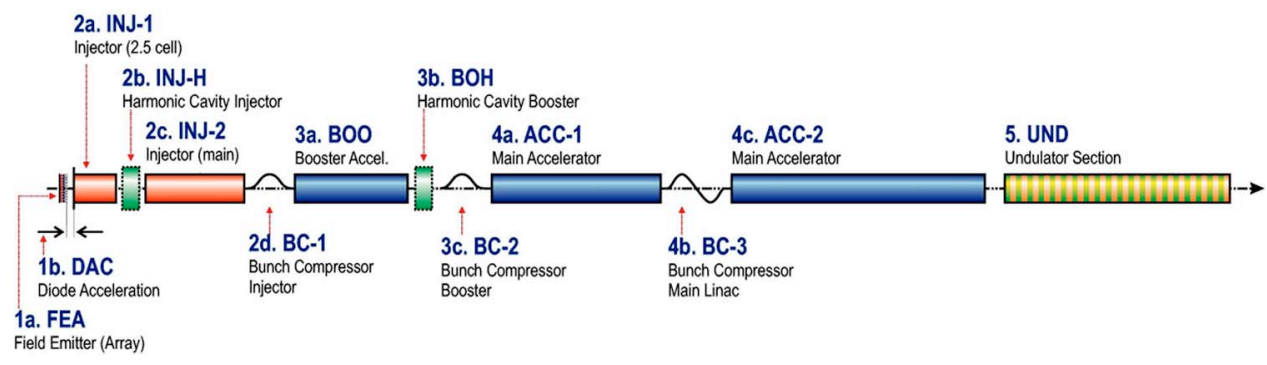

FIG. 1. Generic layout of a FEL.

FEA tips within a millimeter-sized structure resulting extremely large problem sizes. On the other hand, electron/ electron as well as electron/field interactions are very localized, as long as we are looking inside the emitter itself.

The natural approach is to split the problem into modeling the individual emitters in one step and then describing the emission in the global model from an equivalent cathode representing the current and momentum distributions derived from the single emitter calculations.

Within the single emitter calculation, we are trying to also capture real world effects as adsorbents and fabrication tolerances. Currently, we assume FEAs from self-aligning processes, so the dominant effects are current variations due to different field enhancement factors and work functions. These lead to changes in beam size, divergence, and emittance due to nonlinear effects and are transferred into a parametrized model of the emitter.

As a simulation technique, we are using in all cases the particle-in-cell (PIC) algorithm, where instead of Vlasov's equation a coupled model is used. A mesh-based time domain solver handles the electromagnetic field equations and a model using macroparticles solves the equations of motion, where the particles generate currents on the mesh and experience forces due to the field iteratively computed by the solver. The approach is self-consistent, that is, convergent to the real physical behavior within the limits of classical physics.

\section{A. Parametrization of individual emitters}

For rotationally symmetric structures, there is a variety of simulations and simulation codes to compute the beam dynamics. Hartmann et al. ${ }^{2}$ use the boundary element method to solve the potential problem in the emitter geometry and even expanded it to include space-charge effects. Hermannsfeldt et al. $^{3}$ also couple a potential solver (here implemented via finite differences) and a particle tracker to get a consistent solution. Here also magnetic self-fields are included into the simulation. The geometrical resolution of the solver is improved by an iterative grid refinement technique, where they even go down to resolutions of $1-5 \AA$. Both groups omit the effect of the dielectric between emitter and gate in their calculations.

For our purposes, we used the commercial 2.5D code MAFIA TS2D. It is still sufficient to simultaneously resolve nanometer size curvatures at the tip and model the whole emitter region as shown schematically in Fig. 2.
Nonsymmetric emitters such as, e.g., pyramidal tips or asymmetries require the use of a full three-dimensional (3D) treatment of the problem, which could also be handled theoretically by the global solver, described in Sec. II C. Inside the emitter structure, time constants to reach steady state are in the subpicosecond range, so it makes sense to use specialized algorithms assuming static fields for the simulation. One of the options to use would be the MICHELLE code, ${ }^{4}$ which combines a finite-element Poisson solver with a particle tracking algorithm. It is full three dimensional and has a variety of emission models.

In our case, we are using a specialized 3D code developed at the TU Darmstadt, ${ }^{5}$ running also in a multiprocessor mode and using a local grid refinement technique, demonstrated to the left. Starting with a regular grid, the computational cells are recursively split, so that nearly arbitrary grid resolutions can be obtained in the appropriate regions. The method allows for flexible refinement not only at material boundaries, but also in regions of higher particle density and field gradients.

However, specialized numerical techniques are required when dealing with this type of discretization. Because of the nonconforming cells with hanging nodes, which arise when applying local refinement, neither finite differences (FD), nor standard finite elements (FE) approaches can be used in the computation of electrostatic fields. Therefore, a discontinuous Galerkin-FE technique ${ }^{6}$ is adopted in the code for use with nonconforming grids. Given the solution for the electrostatic fields, the space charge is projected into the grid by tracing the fully 3D trajectories of the particles emitted at the emission tip (see Fig. 3). This procedure is repeated until the

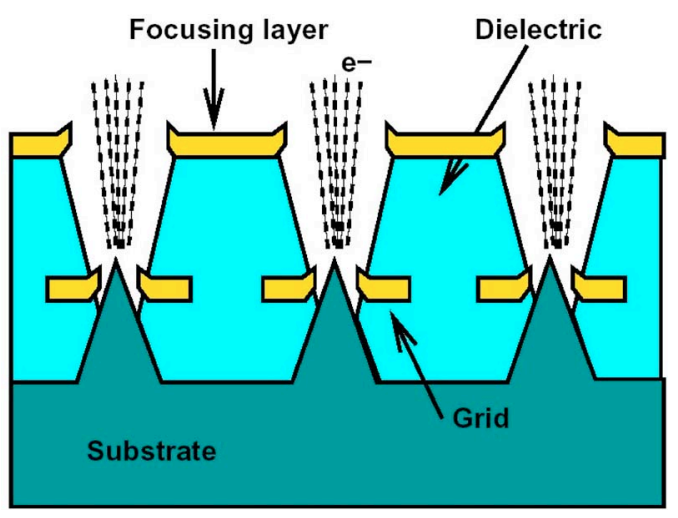

FIG. 2. Field-emitter array with gate and focusing layer. 


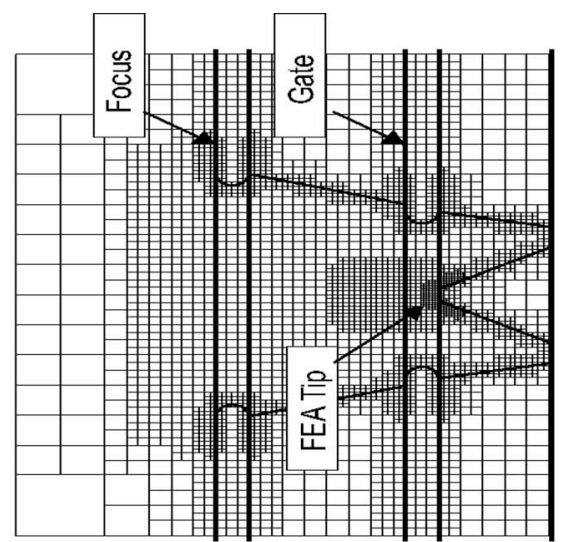

\section{Particle Trajectories}

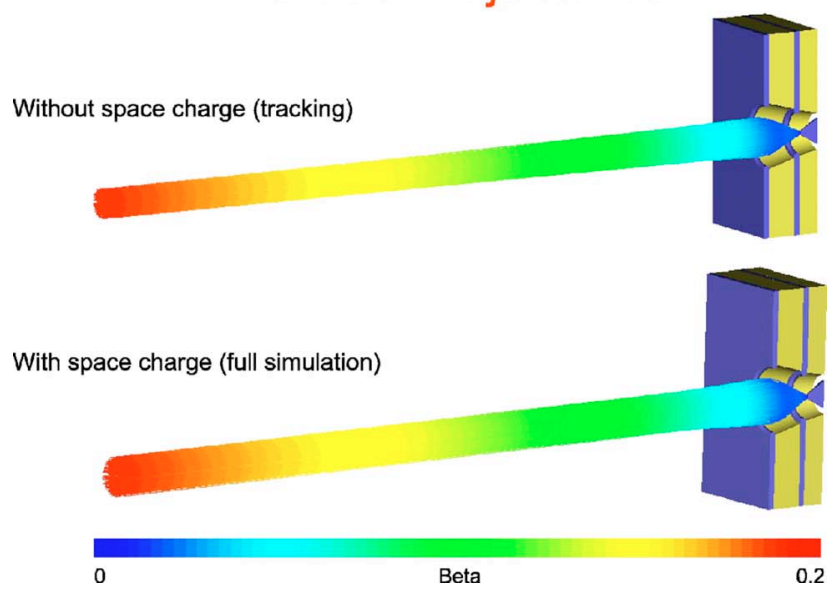

FIG. 3. Geometry discretization with local grid refinement used for the computation of individual emitters. The lower plot shows 3D trajectories with and without space-charge forces.

steady-state space-charge fields and particle trajectories are established. Here, an extremely fine discretization of the emission tip is necessary for resolving the high field gradients, and thus provide for accurate initial conditions in the numerical integration of particle trajectories.

An example of the local refinement used as well as the resulting electron trajectories are shown in Fig. 3. Here, a thirty-two-fold refinement is used allowing for resolutions as fine as $1 \mathrm{~nm}$ in the tip region, while still using only 700000 cells. This resolution is quite comparable with the one used in the two-dimensional simulations with MAFIA TS2 shown below. The computation time of the fully 3D code, however, remains well below $30 \mathrm{~min}$ on a desktop personal computer in single processor mode. Apart from nonrotationally symmetric emitter designs, we are also planning to use it to compute the interaction of neighboring emitters in terms of mutual shielding by space charge.

In the following, we show results obtained for the emitter geometry shown in Fig. 4. Since it is rotationally symmetric, the computation was done with MAFIA TS2.

With a perfectly smooth surface of the emitter, one would need a gate voltage of $600-800 \mathrm{~V}$ to obtain currents in the few hundred microamps region out of a single emitter. As is discussed in Ref. 7, surface roughness in the nanometer

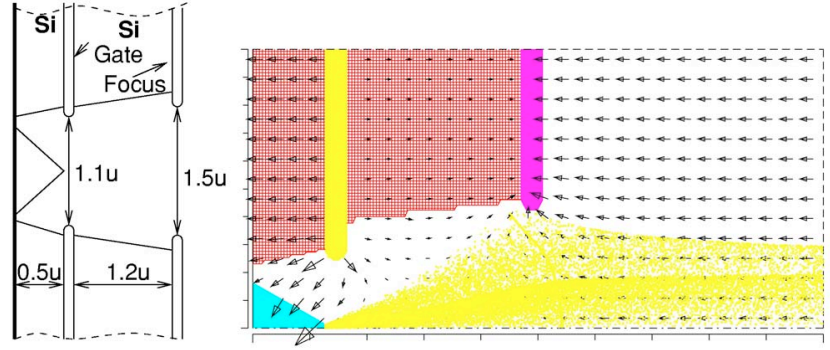

FIG. 4. Rotationally symmetric field-emitter geometry including gate and focusing layer. To the right the electric field together with the particle trajectories are plotted.

range contributes to the field enhancement, so that we can assume an additional factor of 4-5 in the field enhancement to go from the computed peak surface gradient of $1.3 \mathrm{GV} / \mathrm{m}$ to a required value above $5 \mathrm{GV} / \mathrm{m}$.

The voltage at the focusing layer was set to $20 \mathrm{~V}$ to obtain a parallel beam at $0.4 \mathrm{~mA}$. In Fig. 5, the beam radius is plotted as a function of the longitudinal coordinate. As can be expected, higher current leads to stronger space-charge force and thicker beam waists. For high currents, the graphs exhibit a kind of bump at $z=2 \mu \mathrm{m}$. These are actually due to the outer part of the beam hitting the focusing layer and getting absorbed. The power density generated, the fraction of watts over areas with sizes smaller than a micron, is considerable, which would destroy the emitter in continuos operation.

A plot of the phase space distribution for a beam current of $0.4 \mathrm{~mA}$ is shown in Fig. 6. As can be seen, electrons at the outer part of the beam get strongly overfocused, which is due to nonlinearities in the electric field near the edge of the focusing layer.

\section{B. Equivalent emission model}

The next step is to make the transition from individual emitters to a complete cathode including thousands of emission tips. For the global gun simulation, we assume a flat

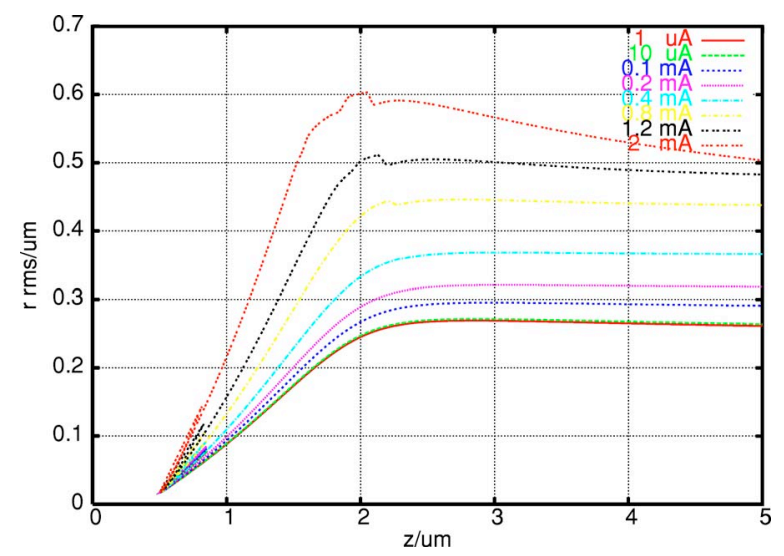

FIG. 5. Evolution of beam radius during flight. The bump in the curves for large currents are due to part of the beam getting dumped into the focusing lens. 


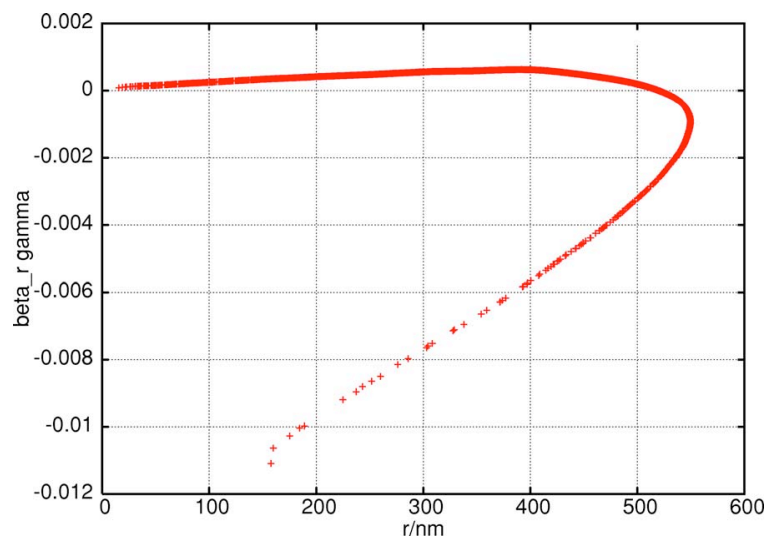

FIG. 6. Normalized phase space distribution of electrons at $0.4 \mathrm{~mA}$ current.

cathode, where emission from an individual tip has been replaced by emission from a flat surface with equivalent current densities and emission angles.

From the results of the previous calculations, we have the working point (gate and focus voltage) and the beam parameters for a design current. Extremely important is to capture the influence of adsorbents and fabrication tolerances on the homogeneity of the emission characteristics. A set of computations with individual tips gives the effect on the resulting beam size, divergence, and emittance, which is used to set up a parametrized model.

The global model uses a given array geometry for the basic distribution, described by array pitch and cathode diameter. Somehow, we have to transfer measurement results of arrays done, e.g., via a scanning anode field-emission microscope (SAFEM) or the density distribution on a phosphorous screen into a stochastic density. The first variables are certainly the mean and standard deviation of the tip currents. Since the emission properties of adjacent tips can be correlated, we also included a correlation length $\tau_{L}$, so that the correlation between tip currents is given as

$$
\frac{1}{\sigma_{I}^{2}}\left\langle I(r), I\left(r^{\prime}\right)\right\rangle=e^{-\left|r-r^{\prime}\right| / \tau_{L}}
$$

Emitter currents in excess of a certain threshold are defined to create a burnout, that is, the tip will not emit at all.

This correlation length even can be related to physical effects. Adsorbents, for example, lead to emission variations, which are completely uncorrelated. This is different from the effect of fabrication tolerances, which may lead to spatial correlations of emission properties of tips within a certain vicinity.

So the geometric distribution of tips over the cathode, stochastic values for the field enhancement function, spatial correlation, and the parametrized emission model coming from the individual emitter calculation together give us a complete equivalent description of a field-emission cathode used for the global gun simulation. Examples for current distributions generated in this way are shown in Fig. 7. Still one has to keep in mind, that these distributions vary at a

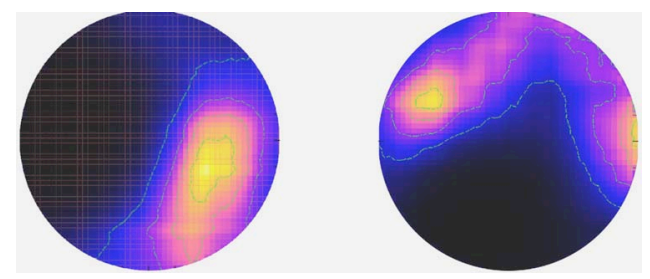

FIG. 7. Example of stochastic current distribution on a $4 \mu \mathrm{m}$ pitch FEA using 17692 tips ( $0.6 \mathrm{~mm}$ cathode diameter). Field enhancement is assumed to be Gaussian distributed with a mean of 10 and a standard deviation of 1 . Variations are spatially correlated over $50 \mu \mathrm{m}$.

micron scale- the global computation has to resolve intrabeam effects at this scale and the computational effort for the total gun is extremely high.

\section{Global structure computation}

As has been indicated, the global structure code CAPONE employs two mutually coupled models, macroparticles to describe the force equations and grid-based fields for Maxwell's equations. Parameter input is done via an extensible markup language (XML) parser package. An additional electrostatic field solver is employed to compute solutions for external accelerating fields. ${ }^{8}$ The modular structure of the code is shown in Fig. 8.

Other than parallelization using shared memory, which is rather easy to implement, message passing via LAM/MPI is used, allowing the program to run on distributed computer networks. The field solver uses a static domain decomposition, where calculation domain patches are distributed among the CPUs. Information about field values at the border between patches are regularly updated between the network nodes.

The macroparticles are distributed dynamically on the CPUs according to position, so that data transfers between particle and field solvers are necessary in order to compute particle forces from the fields and currents from the particle motions do not necessitate any network traffic. As particles cross the boundaries between domain patches, they are getting reallocated to a different CPU.

Nonetheless computation is still dominated by network communication and the parallelization speed up is strongly dependent on the interconnection network used in the cluster. With standard gigabit ethernet, the speed up starts to saturate at around eight CPUs. If today's state of the art, Quadrics QsNetII interconnect is used, even 64 CPUs can be used

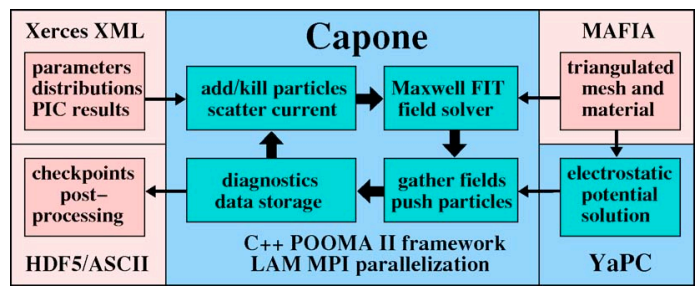

FIG. 8. Functional blocks of massive parallel code for the global gun simulation. 


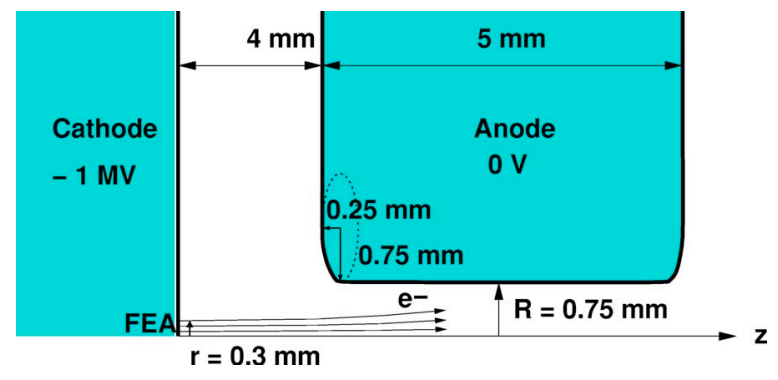

FIG. 9. Geometry of the pulsed dc gun.

giving a speed up by more than a factor 12 compared to the single processor case. The code was used for meshes with up to $10^{9}$ cells resulting in algebraic equations with more than $10^{10}$ unknowns.

\section{RESULTS}

The central figure of merit is the phase space volume occupied by electron described by normalized emittance

$$
\epsilon=\langle\beta\rangle\langle\gamma\rangle \sqrt{\left\langle x^{2}\right\rangle\left\langle x^{\prime 2}\right\rangle-\left\langle x x^{\prime}\right\rangle^{2}},
$$

where $x$ and $x^{\prime}$ are transverse position and flight angle and $\beta$ and $\gamma$ normalized velocity and energy, respectively. Alternatively we can say, that the emittance is the product of uncorrelated momentum spread and size of the emitter. For freeelectron laser (FEL) application, it is not the global emittance which is important, but the value for the center part of the beam, where the current is maximum. Cutting the bunch into slices ( $8.2 \mathrm{fs}$ length) along the longitudinal axis and computing the emittance of the particles in these slices gives the variation along the axis, which we show in the following.

The geometry of the gun, as shown in Fig. 9, is that of a basic diode consisting of two parallel plates with an elliptically rounded iris hole for the beam.

For our preliminary design of a free-electron laser facility, we would need a peak current of $5.5 \mathrm{~A}$. With the momentum spread basically determined by the individual emitters and their stochastic variations, minimizing the emittance means getting that current out of a minimum size emitter. With high-quality processing techniques Schwoebel et al. ${ }^{9}$ give current densities of $38 \mathrm{~A} / \mathrm{cm}^{2}$ of FEA with areas of 7.8 $\times 10^{-3}$ (or $1000 \mu \mathrm{m}$ diameter) and $2 \mathrm{kA} / \mathrm{cm}^{2}$ over areas of $1 \times 10^{-4}$ (or $50 \mu \mathrm{m}$ diameter) in dc operation. In pulsed operation mode, we can expect to push these limits even higher, since the tips will survive temporary overloads, ${ }^{1}$ so we assumed a $600 \mu \mathrm{m}$ diameter FEA. With the individual emitter geometry and parametrization of the preceding sections a $4 \mu \mathrm{m}$ pitch array with roughly 18000 tips, each tip contributing an average of $0.3 \mathrm{~mA}$ was the best compromise leaving still some leeway for variations in the emission current and allowing for dead, nonemitting tips.

Computations were done for different stochastic distributions, which are presented in Fig. 10. Shown are an idealized field-emitter array with perfect emitters, uniform emission from the emitters computed beforehand, and the more real-

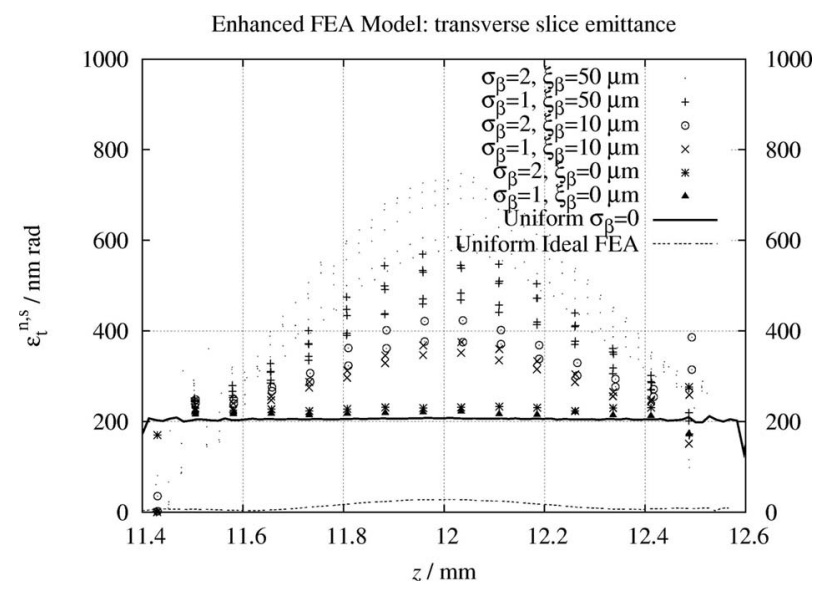

FIG. 10. Emittance distribution inside the bunch for various current distributions. $\sigma_{\beta}$ is the standard deviation of field enhancement factor around a mean of $10 ; \zeta_{\beta}$ is the spatial correlation length.

istic cases, where a Gaussian distribution of the field enhancement factors with different spatial correlation lengths was assumed.

Taking a look at the base line effect, we have a strong contribution to the emittance simply coming from the phase space occupied by the beam directly after emission. Whereas a uniform ideal field-emitter array with perfect parallel beam would give values below $50 \mathrm{~nm}$ rad, we obtain, even without taking into account stochastic variations, a bunch emittance of $200 \mathrm{~nm}$ rad for the FEA modeled. This is beyond our goal value of $50 \mathrm{~nm} \mathrm{rad}$, so further optimization of the emitter geometries has to be done.

Furthermore one sees the effect of inhomogeneities in the current density, assuming the stochastic model described above. It is quite clear, that a larger variation means a worse beam quality, but highly interesting is the effect of the spatial correlation length. In the uncorrelated case, the degradation is really small, only when we have some correlation, resulting in a lumpy current distribution. Here nonlinear intrabeam space-charge forces become much more dominant, giving rise to deformations in the phase space distribution and in turn to a high emittance. Apart from modifying fabrication techniques to minimize these large scale fluctuations, an interesting question is, whether these could be improved by localized conditioning techniques. In a SAFEM-like device, one could think of using a glow discharge between the needle anode of the SAFEM and the FEA to obtain a local surface treatment. Pulse processing similar to Ref. 10, concentrated to narrow regions using the SAFEM needle anode, would also be a very interesting option, but with the shielding effect of the two gates, it is an open question, whether the anode has enough influence on the tip to provoke localized emission.

\section{OUTLOOK}

Via the two-stage approach presented, we are able to do full scale computations of FEA base electron sources. The stochastic emission model has enough degrees of freedom to 
allow a description of most real world effects. The massive parallel code gives the resolution required for the structure.

Currently, we are in the process of modeling synthetic field emitters in order to get a feeling on the systematic behavior. The results are used to find optimum choices for possible field-emitter candidates, specially for emitters currently under development at PSI.

As we are obtaining measurements from a real field emitter by a scanning anode field-emission microscope currently under commissioning at PSI, we are going to be able to calibrate our stochastic emission model allowing to predict overall performances.

${ }^{1}$ R. Ganter et al., Proceedings of the 18th International Vacuum Nanoelectronics Conference IVNC 2005, Oxford, United Kingdom, 10-14 July 2005 (to be published in J. Vac. Sci. Technol. B).

${ }^{2}$ R. L. Hartmann, W. A. Mackie, and P. R. Davis, J. Vac. Sci. Technol. B
12, 754 (1994).

${ }^{3}$ W. B. Hermannsfeldt, R. Becker, I. Brodie, A. Rosengreen, and C. A. Spindt, Nucl. Instrum. Methods Phys. Res. A 298, 39 (1990).

${ }^{4}$ J. J. Petillo, E. M. Nelson, J. F. DeFord et al., IEEE Trans. Electron Devices 52, 742 (2005).

${ }^{5}$ E. Gjonaj and T. Weiland, Proceedings International Workshop on Advances in Computational Multiphysics, Darmstadt, Germany, October 2004 (unpublished)

${ }^{6}$ P. Castillo, B. Cockburn, I. Perugia, and D. Schötzau, Commun. Numer. Methods Eng. 18, 69 (2002).

${ }^{7}$ C. A. Spindt, I. Brodie, L. Humphrey, and E. R. Westerberg, J. Appl. Phys. 47, 5248 (1976).

${ }^{8}$ A. Candel, M. Dehler, M. Troyer, Nucl. Instrum. Methods Phys. Res. A 558, 154 (2006).

${ }^{9}$ LAM/MPI Parallel Computing, http://www.lam-mpi.org

${ }^{10}$ P. R. Schwoebel, C. A. Spindt, and C. E. Holland, Technical Digest of the 17th International Vacuum Nanoelectronics Conference; There has been no accompanying paper to that contribution. In a poster for the 16th International Vacuum Nanoelectronics Conference (again without paper), results were presented for a 100 tip array reaching $1 \mathrm{kA} / \mathrm{cm}^{2}$. 